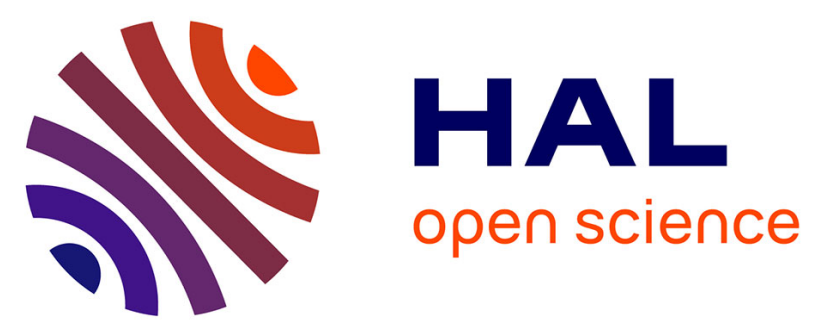

\title{
Measurement of the temperature distribution within monodisperse combusting droplets in linear streams using two-color laser-induced fluorescence
}

Guillaume Castanet, Pascal Lavieille, Michel Lebouché, Fabrice Lemoine

\section{- To cite this version:}

Guillaume Castanet, Pascal Lavieille, Michel Lebouché, Fabrice Lemoine. Measurement of the temperature distribution within monodisperse combusting droplets in linear streams using two-color laserinduced fluorescence. Experiments in Fluids, 2003, 35 (6), pp.563 - 571. 10.1007/s00348-003-0702-1 . hal-01570418

\author{
HAL Id: hal-01570418 \\ https://hal.univ-lorraine.fr/hal-01570418
}

Submitted on 29 Jul 2017

HAL is a multi-disciplinary open access archive for the deposit and dissemination of scientific research documents, whether they are published or not. The documents may come from teaching and research institutions in France or abroad, or from public or private research centers.
L'archive ouverte pluridisciplinaire HAL, est destinée au dépôt et à la diffusion de documents scientifiques de niveau recherche, publiés ou non, émanant des établissements d'enseignement et de recherche français ou étrangers, des laboratoires publics ou privés. 


\title{
Measurement of the temperature distribution within monodisperse combusting droplets in linear streams using two-color laser-induced fluorescence
}

\author{
G. Castanet, P. Lavieille, M. Lebouché, F. Lemoine
}

\begin{abstract}
Two-color laser-induced fluorescence can be use to perform space-averaged flying droplet temperature measurements. In this paper, the possibility to extend this technique to the measurement of the temperature distribution within a moving combusting droplet is considered and demonstrated. This technique may provide new experimental data related to the heat diffusion in liquid fuel droplets injected in high-temperature gas streams, for example, in combustion chambers. The main principles of the technique and the data reduction process are discussed, and a test on combusting a monodisperse ethanol droplets $(200 \mu \mathrm{m}$ in diameter) stream is presented.

\begin{tabular}{ll}
\multicolumn{2}{l}{ Nomenclature } \\
$a_{i}, b_{i}$ & $\begin{array}{l}\text { temperature sensitivity coefficients for } i \text { th } \\
\text { spectral band }\end{array}$ \\
$C$ & molecular concentration of fluorescent tracer \\
$D$ & droplet diameter \\
$I_{0}$ & incident laser beam intensity \\
$I_{f}$ & fluorescence intensity \\
$K_{o p t}$ & optical constant \\
$K_{s p e c}$ & spectroscopic constant \\
$V_{c}$ & collection volume \\
$R_{f}$ & fluorescence ratio \\
$T$ & absolute temperature \\
$T_{i}$ & injection temperature \\
$V_{i}$ & injection velocity \\
$(x, y, z)$ & spatial coordinates
\end{tabular}
\end{abstract}

\section{Greek symbols}

$\beta \quad$ temperature sensitivity coefficient
1

\section{Introduction}

Sprays and droplets are central problems in internal combustion engines. In these kinds of engines, like turbojets or direct-injection diesel engines for automobiles, the fuel is introduced into the combustion chamber by high-pressure atomizers as a liquid spray. A fuel spray consists of a two-phase flow composed of individual droplets that typically range from a few microns to a few hundreds of microns in diameter, with velocities of a tens of milliseconds. When a fuel droplet enters a hightemperature environment, like a combustion chamber, the droplet is heated, evaporates, and finally the fuel vapor burns in order to deliver the energy for propulsion. In the usual combustors of turbojets, the heating phase of the fuel droplet represents about the half of its lifetime in the combustion chamber. Consequently, reliable droplet heating models must be implemented in the predictive calculation tools used by aero-engine designers.

Generally, the experiments on full-scale combustors are complex and rather hard to interpret because of the presence of many highly complex physical phenomena. In order to overcome these problems, idealized sprays are used to improve the basic knowledge in the area of spray combustion. Monodisperse droplet arrays or lines are examples of laboratory experiments that have been widely used in both experimental (Silverman and Dunn-Rankin 1994; Mulholland et al. 1988) and theoretical studies (Chiang et al. 1993).

The understanding of the heating process of a fuel droplet requires basically the measurement of the droplet mean temperature, i.e. the temperature spatially averaged on the droplet volume. Further investigations require the characterization of the temperature distribution within the droplet. A few numerical studies (Chiang et al. 1992; Sazhin et al. 2002) highlighted that the temperature field within a droplet did not match a pure radial heat conduction scheme. These works showed that the heat transfer within a droplet was the result of combined conduction and advection due to the formation of internal vortexes caused by friction phenomena between the liquid droplet surface and the external air flow.

Preliminary measurements of temperature fields of falling droplets were reported by Winter and Melton (1990). The first transient measurements of the temperature distribution within a falling droplet were obtained by $\mathrm{Lu}$ and Melton (2000). The droplets (about $500 \mu \mathrm{m}$ in diameter), initially at ambient temperature, were injected in hot gaseous nitrogen ambient gas, and the time to reach a 
homogeneous temperature field was investigated. In both experiments (Winter and Melton 1990; Lu and Melton 2000), the temperature was measured by the exciplex fluorescence technique, reported in numerous references in the literature (e.g. Muray and Melton 1985; Wells and Melton 1990). However, the experiment was conducted on $500-\mu \mathrm{m}$ diameter droplets, which is not realistic enough for engine spray combustion. Futhermore, the exciplex technique remains very sensitive to oxygen quenching, which constrains this technique to operation under a neutral atmosphere. Consequently, the important case of combusting droplets cannot be investigated. The

two-color laser-induced fluorescence technique, using one single fluorescent tracer and two detection spectral bands, was developed by Lavieille et al. (2001a, 2002) to measure the mean droplet temperature in a monodisperse droplet stream. The main advantages of the two-color laserinduced fluorescence technique are the total independence of the measurement on the measuring volume, i.e. the fraction of the droplet illuminated by the laser excitation, the local laser intensity and the tracer concentration. This paper provides an extension of this technique to the measurement of the temperature distribution within a droplet by scanning the droplet volume by a sufficiently small probe volume compared to the droplet volume itself. A model based on the $3 \mathrm{D}$ refraction law allows one to properly find the position of the probe volume in the droplet because of laser beam refraction phenomena at the air-liquid interface. A suitable data reduction process allows one to construct a temperature map in an equatorial plane of the droplet. The paper describes the experimental set-up and the data reduction strategy in order to map the temperature inside the droplet. Experimental results were obtained on a combusting monodisperse ethanol droplet stream (droplet diameter of about $200 \mu \mathrm{m}$ ). The distribution of the temperature was determined at different times, and the convective heat transfer phenomena inside the droplet were clearly highlighted.

\section{2}

\section{Principles of two-color laser-induced fluorescence applied to mean droplet temperature measurements}

\section{1}

\section{Principles}

Although the main features of two-color laser-induced fluorescence technique are detailed in this section, further technical details can be found in a previous paper (Lavieille et al. 2001a). The fuel, ethanol here, is previously seeded with a low concentration (a few $\mathrm{mg} / \mathrm{l}$ ) of rhodamine $\mathrm{B}$. Rhodamine $B$ is an organic dye usually used as fluorescent temperature sensor. Furthermore, the fluorescence of rhodamine $\mathrm{B}$ can be easily induced by the green line $(\lambda=514.5 \mathrm{~nm})$ of the argon ion laser and is a suitable candidate to be used as a temperature tracer (Copetta and Rogers 1998). The rhodamine B fluorescence spectrum is broadband, and it has been shown that its temperature sensitivity is strongly dependent on wavelength (Lavieille et al. 2001a). The fluorescence intensity expression at the wavelength $\lambda$ as a function of the different physical and optical parameters is given by (Lemoine et al. 1999; Lavieille et al. 2001a)

$I_{\mathrm{f}}(\lambda)=K_{\mathrm{opt}}(\lambda) K_{\text {spec }}(\lambda) V_{\mathrm{c}} I_{0} \mathrm{Ce}^{\beta(\lambda) / T}$,

where $K_{\text {opt }}$ is an optical constant, $K_{\text {spec }}$ is a constant that depends solely on the spectroscopic properties of the fluorescent tracer in its environment (i.e. the fuel), $I_{0}$ the laser excitation intensity, $C$ the molecular tracer concentration, $T$ the absolute temperature, and $V_{c}$ the collection volume of the fluorescence photons. The product $C \cdot V_{\mathrm{c}}$ of the collection volume by the tracer molecular concentration is, in fact, related to the number of fluorescence photons emitted by the rhodamine B molecules excited by the laser radiation and reaching the photodetector surface. This parameter is strongly related to the droplet size and to the probe volume dimensions. The factor $\beta(\lambda)$ characterizes the temperature dependence of the fluorescence intensity at the wavelength $\lambda$.

To collect a sufficient number of photons, the signal is detected on a spectral band $\left[\lambda_{i 1} ; \lambda_{i_{2}}\right.$ ], where $i$ denotes the spectral band. The resulting signal can be expressed by using a second-order expression

$$
\begin{aligned}
I_{\mathrm{f} i} & =\int_{\lambda i 1}^{\lambda i 2} K_{\mathrm{opt}}(\lambda) K_{\mathrm{spec}}(\lambda) V_{\mathrm{c}} I_{0} C \mathrm{e}^{\beta(\lambda) / T} \mathrm{~d} \lambda \\
& \approx K_{\mathrm{opt} i} K_{\text {spec } i} V_{\mathrm{c}} I_{0} C \mathrm{e}^{\frac{\mathrm{a}_{i}}{T}+\frac{b_{i}}{T^{2}}}
\end{aligned}
$$

where $K_{\mathrm{opt} i}$ and $K_{\text {speci }}$ are the optical and spectroscopic constants related to the spectral band $i$. The parameters $a_{i}$ and $b_{i}$ are the temperature sensitivity coefficients for the spectral band $i$. In many works, the second-order term is skipped, the first-order being sufficient, except for large temperature variations. In order to properly measure the temperature of a moving and combusting droplet, the influence of the parameters $C \cdot V_{\mathrm{c}}$ and $I_{0}$ must be removed. In combustion situations, the dye concentration is also likely to vary, and the collection volume constantly changes as the droplet crosses the probe volume. Furthermore, the intensity distribution of the laser excitation within the droplet depends on the position of the droplet because of the lensing effect of the droplet interface. In order to remove these problems, the fluorescence intensity is detected on two spectral bands for which the temperature sensitivity is highly different. The selection of these spectral bands is optimized by an initial study of the fluorescence spectrum against temperature (Lavieille et al. 2001a). The fluorescence ratio between both fluorescence intensities collected on both optimal spectral bands is given by

$R_{\mathrm{f}}=\frac{I_{\mathrm{f} 1}}{I_{\mathrm{f} 2}}=\frac{K_{\mathrm{opt1}}}{K_{\mathrm{opt} 2}} \frac{K_{\text {spec } 1}}{K_{\text {spec } 2}} \mathrm{e}^{\frac{a_{1}-a_{2}}{T}+\frac{b_{1}-b_{2}}{T^{2}}}$.

This ratio is totally independent of the dimensions of the intersection between the droplet, the laser excitation volume and the photon collection volume. The influence of the local laser excitation intensity and tracer concentration are also totally eliminated. The use of a single reference point where the temperature is known allows us to eliminate the optical and spectroscopic constants. The resulting temperature sensitivity is about $2 \% / \mathrm{K}$. 


\section{2}

\section{Optical parameters}

According to the preliminary spectral studies (Lavieille et al. 2001a), the following two spectral bands were selected because it is the better trade-off for optimizing the temperature sensitivity:

- band 1: center wavelength $\lambda_{c}=530 \mathrm{~nm}$, bandwidth, $\Delta \lambda=10 \mathrm{~nm}$

- band 2: $\lambda>590 \mathrm{~nm}$ (high-pass filter)

The result is that a $1{ }^{\circ} \mathrm{C}$ temperature variation corresponds to about a $2 \%$ variation in the fluorescence ratio.

The influence of the optical paths was characterized in order to justify the optically thin conditions approximation performed in Sect. 2.1. The absorption of the fluorescence itself is strongly different in the two selected color bands, since the first one coincides roughly with the maximum of the absorption spectrum. The measured absorption coefficients for the fluorescence on the two selected spectral bands are (Lavieille et al. 2001a):

- $\varepsilon_{\mathrm{f} 1}=1.18 \times 10^{7} \mathrm{~m}^{-1} \mathrm{~mol}^{-1} 1$ for $\lambda \in[525 \mathrm{~nm}, 535 \mathrm{~nm}]$ at $T=22^{\circ} \mathrm{C}$,

- $\varepsilon_{\mathrm{f} 2}=1.5 \times 10^{6} \mathrm{~m}^{-1} \mathrm{~mol}^{-1} \mathrm{l}$ for the band $\lambda>590 \mathrm{~nm}$ at $\mathrm{T}=22^{\circ} \mathrm{C}$.

The coefficient $\varepsilon_{\mathrm{f} 1}$ appears 8 times higher than $\varepsilon_{\mathrm{f} 2}$, which may have serious consequences on fluorescence reabsorption and may greatly change the fluorescence ratio in the case of large optical paths. However, for a 200$\mu \mathrm{m}$ droplet diameter, the fluorescence reabsorption should not exceed $1 \%$ (i.e. a temperature deviation of $0.5^{\circ} \mathrm{C}$ ), in the worst case, for the rhodamine $\mathrm{B}$ concentration used $\left(\mathrm{C}=2 \cdot 5 \cdot 10^{-6} \mathrm{~mol} / \mathrm{l}\right)$.

\section{3}

\section{Principles of combustion experiment of monodisperse droplets}

A linear monodisperse droplet stream is generated by Rayleigh disintegration of a liquid jet, with the use of a mechanical vibration obtained by a piezoceramic excited by a square wave. The applied voltage on the piezoceramic depends on the desired position of the break-up zone and on the physical properties of the fuel related to the injection temperature. For a given frequency, the liquid jet breaks up into equally spaced and monosized droplets at the frequency of the forced mechanical vibration. The liquid fuel is pressurized with compressed air between 0.2 and 0.6 bars and is forced through a calibrated orifice ranging from $\Phi_{0}=50 \mu \mathrm{m}$ to $\Phi_{0}=100 \mu \mathrm{m}$ in diameter. According to the Rayleigh theory, the resulting droplet diameter is about $1.9 \Phi_{0}$. For the present experiment, a $100-\mu \mathrm{m}$ hole diameter was used. The droplet injection velocity can range from $2 \mathrm{~m} / \mathrm{s}$ to about $10 \mathrm{~m} / \mathrm{s}$. The fuel can be preheated in the injector body by means of an external heated water circulation system. The temperature of the fuel is measured exactly at the injection point with the use of a K-type thermocouple. An electrically heated coil allows us to initiate the combustion just after the break-up zone of the liquid jet and a laminar flame appears (Fig. 1).

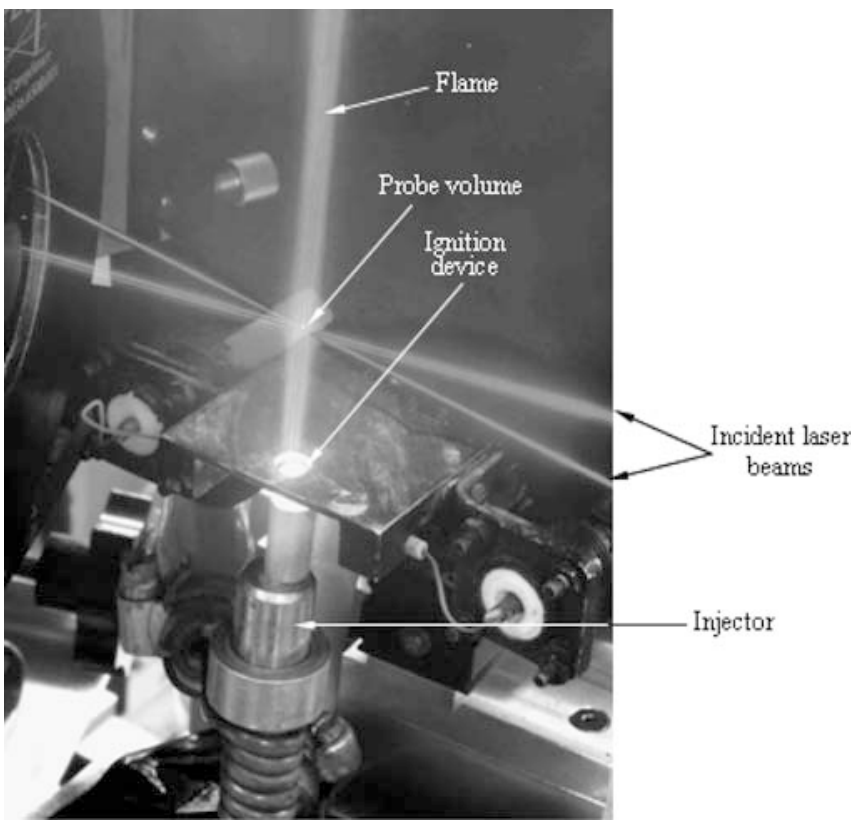

Fig. 1. Picture of the injector, with the monodisperse droplet stream in combustion

\section{4}

\section{Optical set-up}

The optical set-up can be divided into two parts: the emission optics and the reception set-up. The reception optics includes the collection optics and the set-up that divides the fluorescence intensity into the two selected spectral bands and processes the signals.

\section{1}

\section{Emission optics}

A LDV optical device, providing two intersecting laser beams issuing from the same laser source, was used in order to create the probe volume (Fig. 2). On the one hand, a mean droplet temperature can be obtained by using a probe volume diameter comparable to the droplet diameter itself and by averaging the fluorescence signal on the overall transit time of the droplet in the probe volume (Lavielle et al. 2001a, 2002).

In the other hand, mapping the temperature field within a $200-\mu \mathrm{m}$ diameter droplet requires the use of a particularly small probe volume. The probe volume effective size is given by the combination of the laser excitation area and the receiving optics resolution. The combination of a beam expander and a short focal front lens for the LDV system allows one to obtain an ellipsoidally shaped laser excitation volume, with the dimensions $20 \times 20 \times 90 \mu \mathrm{m}$ (Fig. 3 ). In the present experiment, the main advantage of using a LDV optical setup are:

- The measured droplet velocity enables us to determine the time elapsed from the injection point. Inversely, the position of the probe volume in the droplet can be accurately calculated with the use of the measured velocity if the probe volume diameter is sufficiently small compared to the droplet. 

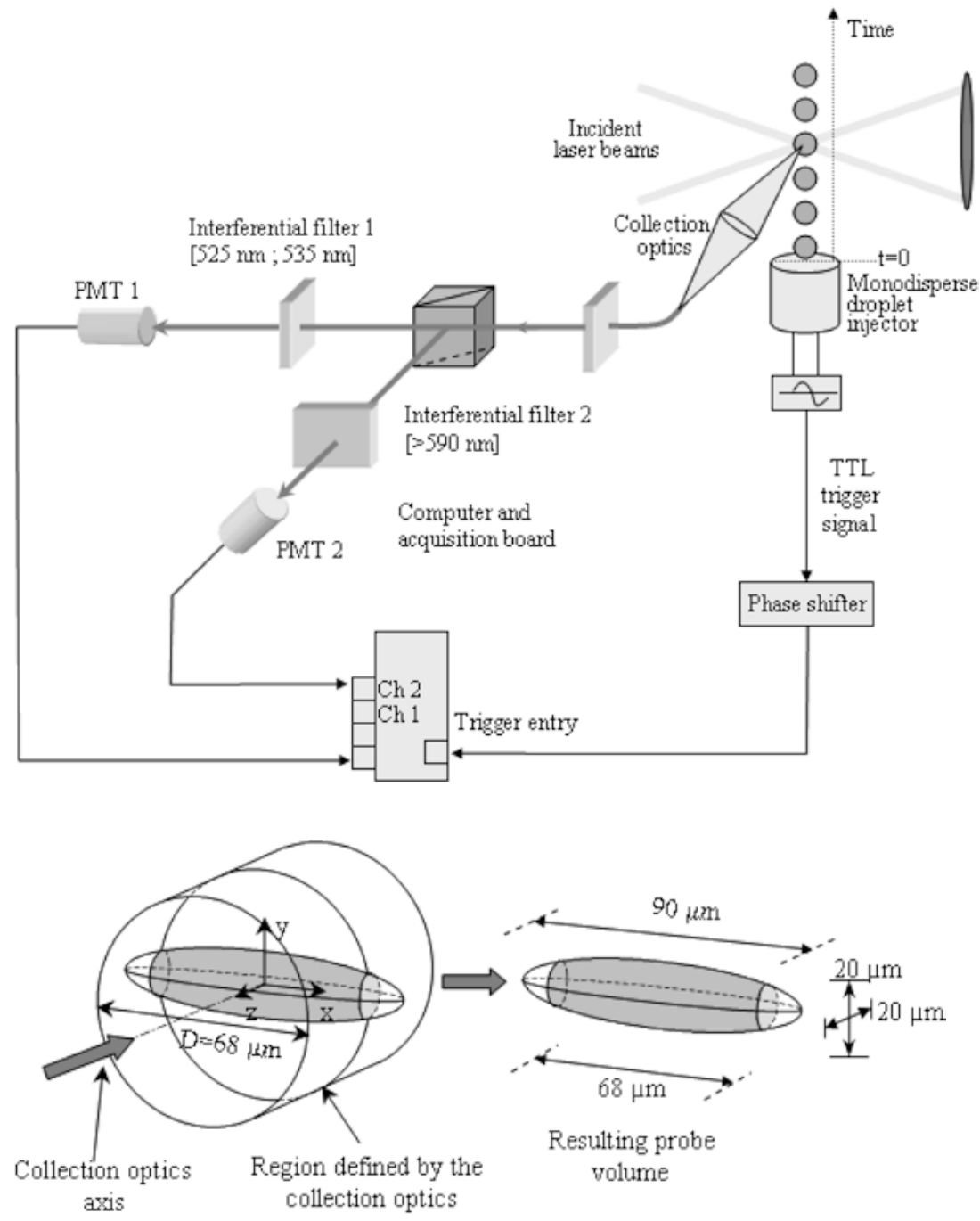

Fig. 2. Block diagram of the experimental set-up

Fig. 3. Definition of the probe volume

- This kind of optical configuration provides a localized high-intensity laser excitation area, which allows us to perform spatially localized measurements.

\section{2}

\section{Reception optics}

The fluorescence signal is collected at a right angle by means of an achromatic doublet connected to an optical fiber, which acts as a pinhole. According to the selected focal length for the fluorescence signal receiver, the collection volume corresponds to a $68-\mu \mathrm{m}$ diameter cylinder, assuming that the depth of field is on the order of the laser beam diameter. Furthermore, the lensing effect at the droplet surface reduces this size from $68 \mu \mathrm{m}$ to $57 \mu \mathrm{m}$ when the intersection point of the laser beams is located in the droplet center. The resulting probe volume dimensions are roughly $20 \times 20 \times 57 \mu \mathrm{m}$ (Fig. 3 ), which is sufficiently small compared to the droplet diameter. Therefore it becomes possible to perform temperature measurements within the droplet. The fluorescence signal is transmitted by the optical fiber to a set of beamsplitters and optical filters, which enables us to divide the fluorescence signal into the two specified spectral bands, as indicated in Sect. 2.2 (see also Lavieille et al. 2001a). The laser light scattering on the droplets is eliminated by the use of a filter (Super Notch Plus, Kaiser Optical) with optical density 6 at $\lambda=514.5 \mathrm{~nm}$. The fluorescence signal is detected on the two spectral bands by means of two photomultiplier tubes (Hamamatsu R2066) equipped with two rapid preamplifiers (10-MHz bandwidth).

\section{5}

\section{Principles of droplet temperature mapping and data reduction}

\section{1}

\section{Principles of droplet temperature mapping}

As a droplet crosses the laser excitation area in the stream direction, if the probe volume is sufficiently small compared to the droplet, different zones of the droplet are successively illuminated by the laser and emit fluorescence, which can be processed with the two-color technique. By shifting the probe volume in a perpendicular axis of the stream direction, it is possible to scan different lines in the droplet. The different axes related to the localization of the probe volume in the droplet are represented in Fig. 4. The droplet moves along the $y$-axis, and the fluorescence is collected at a right angle on the $x$-axis. The bisecting line of the two laser beams defines the 


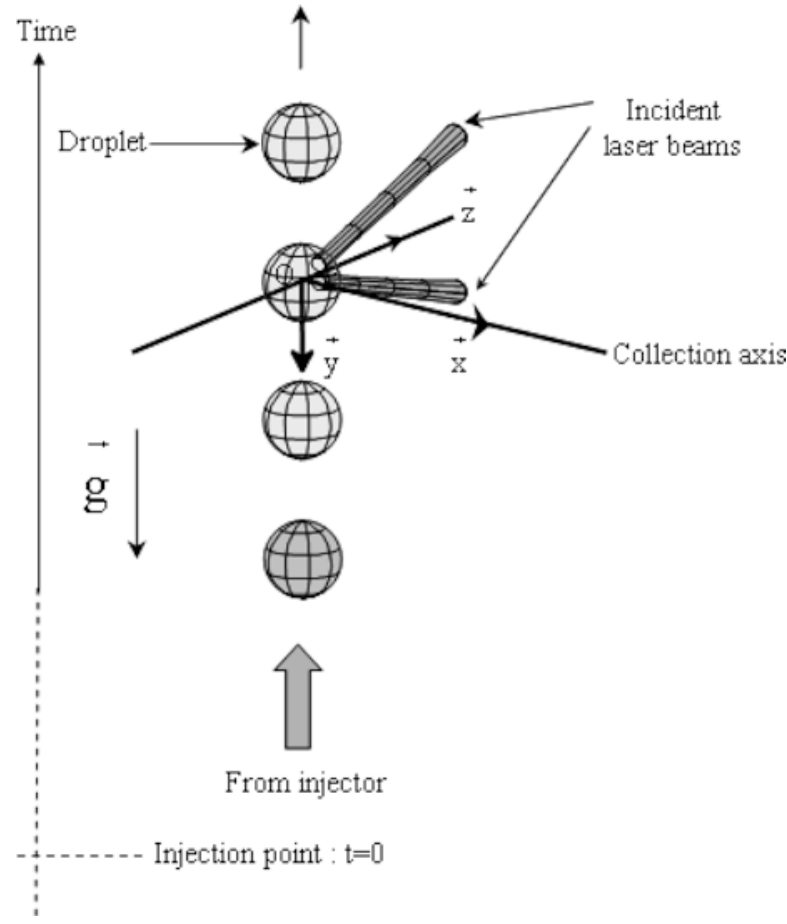

Fig. 4. Definition of the axis

$z$-axis, which is perpendicular to $x$ and $y$. The laser beams are therefore located in the $\mathrm{y}$ - $\mathrm{z}$-plane in absence of refraction caused by the droplet interface.

The position of the droplet in the fixed $x-y-z$ coordinate system is then determined by:

- the $y$-coordinate along the stream axis calculated by means of the measured droplet local velocity, which corresponds to the natural scanning of the droplet along the stream axis

- the $x$-coordinate, which defines the distance between the stream axis and the measurement line along the $y$-axis

- the $z$-coordinate, which remains fixed at $z=0$ in the present case

The point ( $x=0, y=0, z=0$ ) corresponds to positioning the intersection of the laser beams at the droplet center.

The droplet axis of symmetry is determined initially by the localization of the axis of symmetry of the fluorescence signal. The signal is spatially averaged on the intersection between the laser excitation volume and the volume defined by the collection optics. A set of illustrations shows the intensity distribution in an $x-z$-plane and the collection optics volume projection in this plane in Figs. $5 \mathrm{a}-\mathrm{c}$, where Fig. $5 \mathrm{a}$ is the centered situation $(x=0, y=0$, $z=0$ ), and Figs. $5 \mathrm{~b}$ and $5 \mathrm{c}$ are off-centered situations at $(x=0, y=65, z=0)$ and $(x=45, y=-40, z=0)$ respectively. In all these pictures, the local incident intensity distribution in the probe volume was calculated by the model described in Lavieille et al. (2000), which takes into consideration the Gaussian distribution of energy in the laser beam and the lensing effect of the droplet interface. The $3 \mathrm{D}$ refraction at the droplet interface and that of the laser beam axis and of the collection volume are also
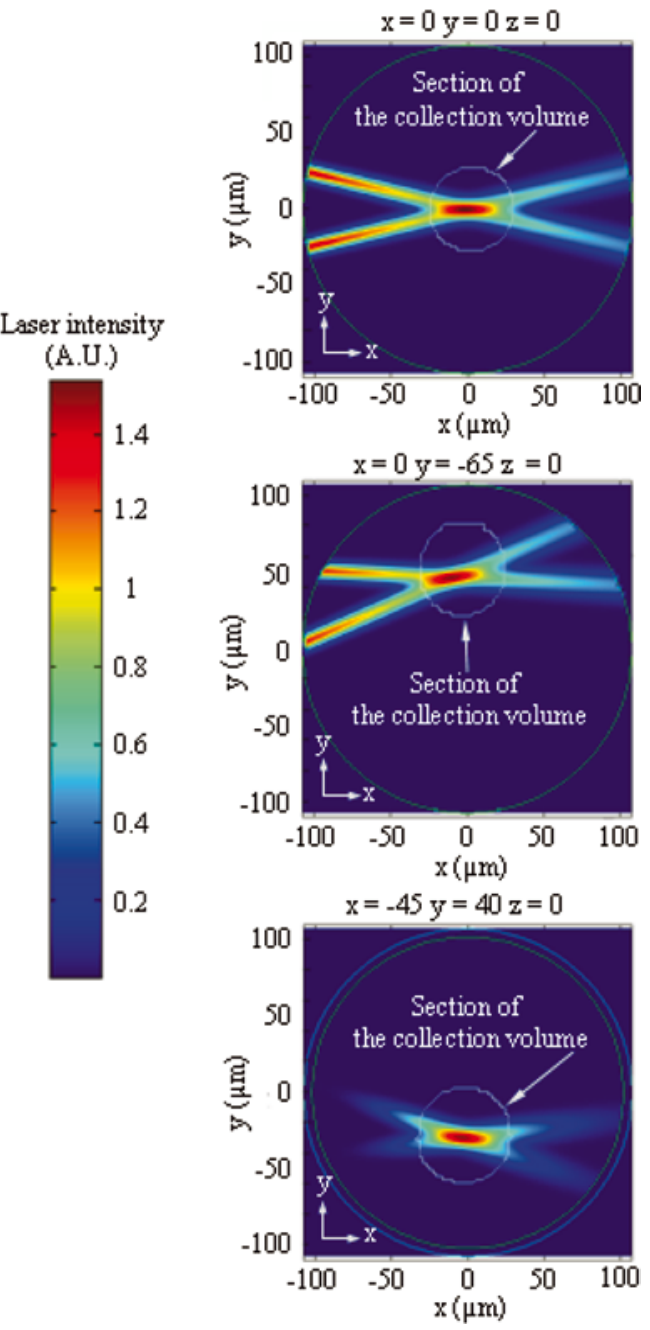

Fig. 5. Distribution of the excitation intensity in the plane $(x, y)$ containing the intersecting point of the laser beam axis

included in this model. In this set of figures, it can be clearly seen that the main area contributing to the fluorescence signal is located around the intersection point of the beam axis. Furthermore, it can be checked that this point is always in the field of view of the collection optics, regardless of the position of the droplet in the $x-y$-plane.

\section{2}

\section{Data acquisition and reduction}

The scanning time of the droplet by the probe volume is very short; for example, this time is about $30 \mu$ s for a 200 $\mu \mathrm{m}$ diameter droplet streaming at $8 \mathrm{~m} / \mathrm{s}$. Consequently, an accurate description of the fluorescence distribution requires a high sampling frequency. An acquisition board enables us to sample the fluorescence signal simultaneously on two channels at $20 \mathrm{MHz}$ with 12-bit resolution for the A/D conversion. Consequently, a measurement line on the $y$-axis (at $x=0$ and $z=0$ ) is described by about 600 samples. Because of the random process of the photons arrival on the photocathode of both PM tubes, noticeable noise appears in the space distribution of the fluorescence signal. This phenomenon is well known in the fluorescence detection process as a Poisson noise (Gaskey et al. 1990). 
The periodicity and the repeatability of the droplet trajectories allow us to process phase-locked averaged measurements on a suitable number of droplet transits in the probe volume. As illustrated in the block-diagram of Fig. 2, a TTL trigger signal is generated at each droplet emission by the injector at the piezoceramic frequency. This signal is then phase-shifted in order to take into account the elapsed time between the measuring position and the injection point. The fluorescence signal distribution corresponding to the scanning of the droplet in the streaming direction is then phase-locked and averaged on about 1000 droplet transits. The same work is performed on both acquisition channels, corresponding to the acquisition in both color bands (Fig. 6). The fluorescence ratio is calculated at each measurement point after completion of the averaging process (Fig. 6), and the temperature is determined by relation (3). To construct a temperature map, this process is repeated at different $x$-positions separated by 10 to $20 \mu \mathrm{m}$, depending on the droplet diameter. The lateral spread of the droplet trajectories is estimated to be less than $10 \mu \mathrm{m}$ by observing the droplet signal variations in the vicinity of the droplet border. This limits the distance of investigation from the injection point, since the turbulent motions of the flame tend to increase the trajectory spread as the distance from the injection point is increased.

The 3D refraction laws are used in order to accurately determine the deviation of the laser beams axis at the droplet interface and to position their intersecting point, and subsequently the real location of the measurement point within the droplet. A constant refractive index for ethanol at $20^{\circ} \mathrm{C}(n=1.36)$ was considered in these experiments, in spite of the expected presence of temperature gradients inside the droplet. The simple 3D refraction law applied at the spherical interface cannot enable us to position all the measurement points within the droplet volume, since the points located near the droplet surface are in a total reflection situation. Consequently, only a part of the droplet volume can be mapped. Figure 7 shows a 3D view of the measurement points positioned in the droplet volume, after the conversion of the fluorescence ratios into temperature. The $3 \mathrm{D}$ profiles can be converted into a planar map of temperature in a droplet equatorial plane by

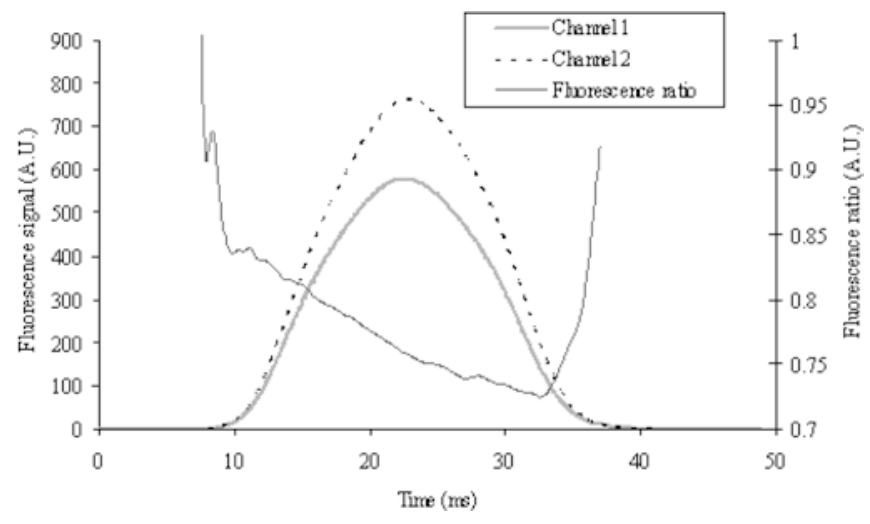

Fig. 6. Example of fluorescence signal acquisition on the two color bands and calculation of the fluorescence ratio, on a particular position, averaged on the transit of 1000 droplets

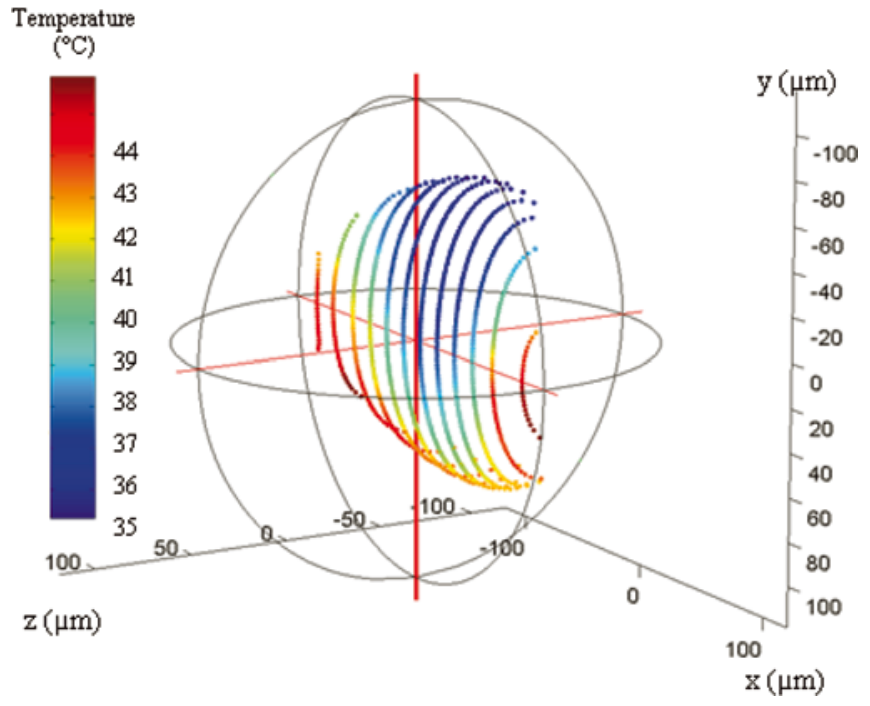

Fig. 7. Temperature measurements on the $y$-axis, at different $x$-positions used to construct a temperature map (case of the combusting droplet, $t=9.6 \mathrm{~ms}$ )

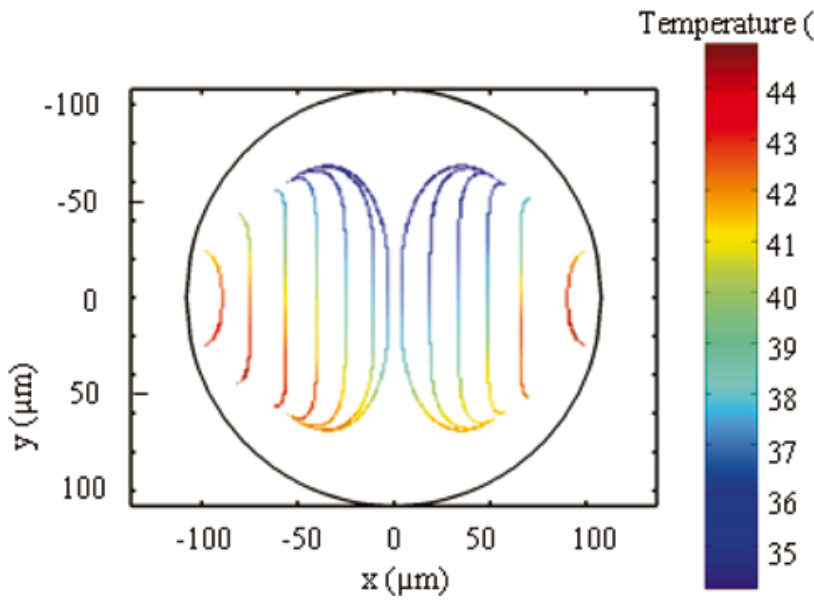

Fig. 8. Temperature measurements on the $y$-axis, at different $x$-positions, positioned in an equatorial plane using the symmetry properties (case of the combusting droplet, $t=9.6 \mathrm{~ms}$ )

using the axisymmetry properties (Fig. 8). Finally, a linear interpolation of the temperature values between the different points positioned in the equatorial plane is calculated in order to obtain a continuous temperature map.

\section{6}

\section{Preliminary tests}

\section{1}

Calibration of the temperature response

The fuel (ethanol) for the combustion tests was prepared as recommended by Lavieille et al. (2001b), with a dye concentration $C=2.5 .10^{-6} \mathrm{~mol} / \mathrm{l}$ (rhodamine B) and a large excess of sodium hydroxide $(\mathrm{NaOH})$ in order to stabilize the rhodamine $\mathrm{B}$ in a basic medium. This prevented the acidification process of the liquid ethanol during the combustion, since the rhodamine B fluorescence spectrum evolves with the $\mathrm{pH}$ value in an acid medium. In this way 
the fluorescence ratio becomes insensitive to any changes in the composition of the liquid phase: this has been demonstrated by taking samples of partially burnt droplets of ethanol in the combusting jet (Lavieille et al. 2001b). A calibration of the fluorescence emission induced by the green line of the argon ion laser $(\lambda=514.5 \mathrm{~nm})$ in the two selected spectral bands was performed for this fuel in order to determine the temperature sensitivity coefficient set $\left(a_{1}-a_{2}\right)$ and $\left(b_{1}-b_{2}\right)$ in Eq. (3). A glass-wall cell was filled with the fuel and was progressively heated by a resistor. The fuel was constantly agitated in order to avoid photobleaching effects and to enable quick thermal homogenization. The fluorescence signal was collected very close to the window in order to avoid fluorescence reabsorption phenomena, which are likely to give erroneous fluorescence ratios (Lavieille et al. 2001a), and the temperature was recorded by means of a thermocouple. Finally, the temperature sensitivity coefficient was calculated by plotting the quantity $\ln \left(R_{\mathrm{f}}\right)$ as a function of $(1 / T)$ (Fig. 9). The polynomial fit of the calibration curve gives $a_{1}-a_{2}=-4462.9 \mathrm{~K}$ and $b_{1}-b_{2}=5.524 \times 10^{5} \mathrm{~K}^{2}$.

\section{2}

\section{Preliminary validation of the technique}

In order to test the validity of the technique, a first set of experiments for a jet operated under quasi-isothermal conditions was performed. The droplets (diameter at injection: $185 \mu \mathrm{m}$ ) were injected at the ambient temperature, without combustion in the thermal boundary layer of a vertical heated plate, in order to compensate for cooling by evaporation. The goal was to obtain, as much as possible, gradient-free ethanol droplets. An other possibility was to inject ethanol droplets in a vapor-saturated enclosure, but such a device was not available. In the investigated area, the mean droplet temperature evolved from $22.6^{\circ} \mathrm{C}$ to $23.7^{\circ} \mathrm{C}$ in $1.7 \mathrm{~ms}$ : the droplets are almost isothermal and moderate temperature gradients are expected within the droplets. The mapping technique, described in the last section, was implemented at an intermediate step between $22.6^{\circ} \mathrm{C}$ and $23.7^{\circ} \mathrm{C}$. In view of the results, the fluorescence ratio appeared almost homogenous in all the $\mathrm{x}$-y-equatorial planes. The fluorescence ratio map was

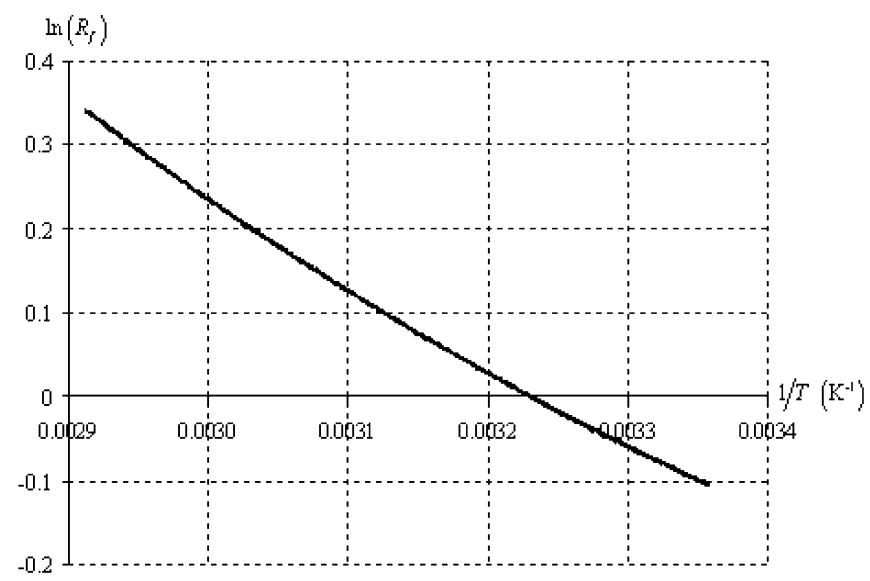

Fig. 9. Calibration curve of rhodamine B dissolved in the fuel (ethanol+ $\mathrm{NaOH}$ at $C=10^{-3} \mathrm{~mol} / \mathrm{l}$ ) converted into a temperature map (Fig. 10). As predicted, all the measurement points could not be positioned due to the total refection phenomena, as previously mentioned (see Fig. 8). The symmetry of the temperature map, in respect to the streaming axis, can be noted. A cold area can be seen in the vicinity of the droplet head, corresponding to a zone cooled by the vaporization of the ethanol. The presence of heat diffusion from the gaseous medium of the heated bench can also be observed on a moderate area of the right and left borders of the droplet. For the remaining area of the droplet, i.e. $x$ ranging from $-70 \mu \mathrm{m}$ to $70 \mu \mathrm{m}$ and $y$ from $-20 \mu \mathrm{m}$ to $60 \mu \mathrm{m}$, the temperature appears quite homogeneous, and the temperature variations do not exceed $1.5^{\circ} \mathrm{C}$. This is clearly a good estimation of the temperature random error in the mapping process.

\section{7}

\section{Experimental results in combustion}

The technique was tested in combustion conditions in the phase of the intense heating of the droplets. Monodisperse droplets of initial diameter $216 \mu \mathrm{m}$ were preheated in the injector body in order to make the inflammation easier. The injection conditions are $T_{\mathrm{i}}=38^{\circ} \mathrm{C}$ and $V_{\mathrm{i}}=9.2 \mathrm{~m} / \mathrm{s}$. The combustion is initiated $30 \mathrm{~mm}$ after the injector exit. The mean temperature evolution of a droplet in a flame has already been characterized (Lavielle et al. 2001a, 2001b). A first phase corresponds to the intense heating of the droplet by forced convection because of the high level of the background temperature. At the end of this phase, the droplet temperature attains an equilibrium temperature corresponding to a wet bulb temperature, below the boiling temperature of ethanol $\left(78^{\circ} \mathrm{C}\right)$. During the equilibrium phase, the droplets vaporize at constant temperature.

Because of the sharp temporal evolution of the mean droplet temperature in the heating phase (Fig. 11), strong temperature gradients within the droplet are expected. The droplet velocity, measured by LDV, used for the calculation of the time elapsed from the injector is also reported in Fig. 11. The slight decrease in the velocity is related to

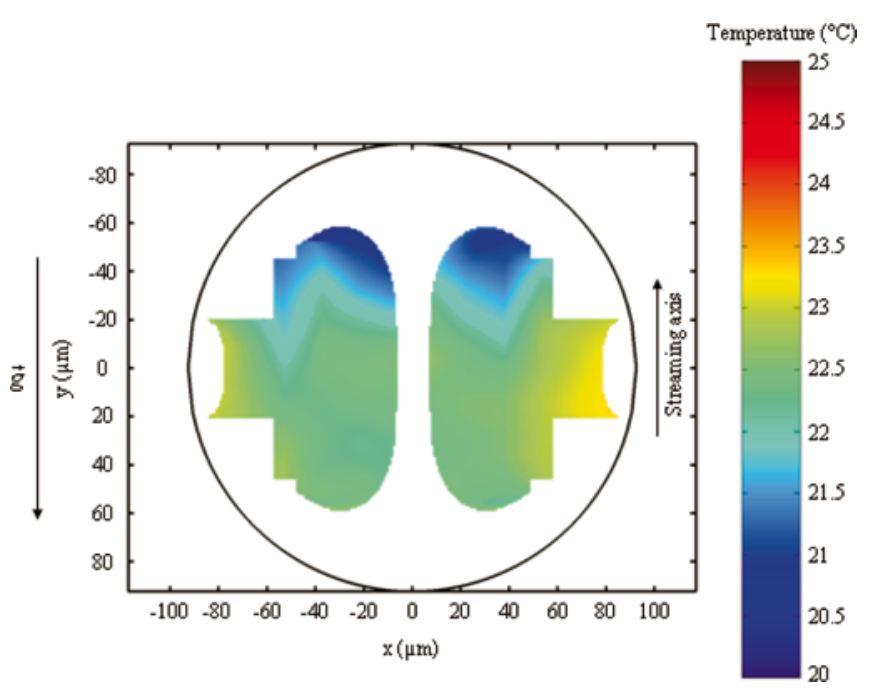

Fig. 10. Construction of a temperature map under quasi-isothermal conditions 


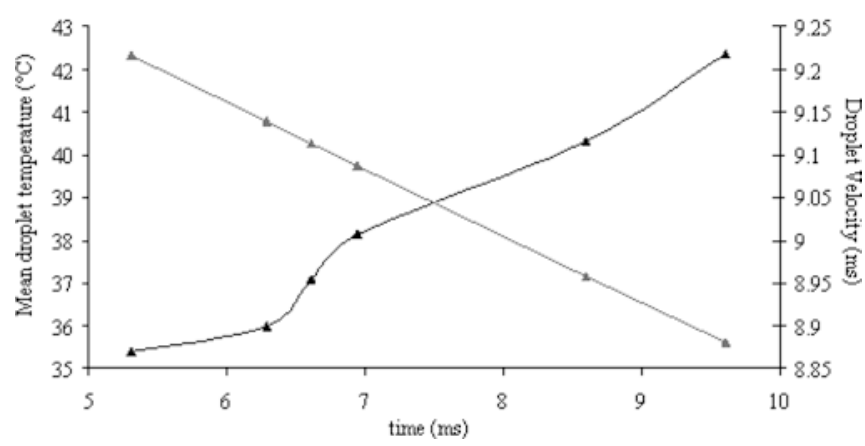

Fig. 11. Mean droplet temperature and velocity evolution in the flame as a function of time (blacktriangle $=$ Temperature ${ }^{\circ} \mathrm{C}$; grey triangle $=$ Velocity $\mathrm{ms}$ )

the effect of the drag forces (Poo and Ashgriz 1991). Temperature maps were constructed at $50 \mathrm{~mm}, 59 \mathrm{~mm}$, $62 \mathrm{~mm}, 65 \mathrm{~mm}, 80 \mathrm{~mm}$ and $89 \mathrm{~mm}$ from the injection point, i.e. between the instants $t=5.3 \mathrm{~ms}$ and $t=9.6 \mathrm{~ms}$, where $t=0$ was taken at the injector exit. The droplet diameter reduction because of evaporation was evaluated between $t=5.3 \mathrm{~ms}$ and $t=9.6 \mathrm{~ms}$, with the help of the experimental results of Virepinte et al. (2000) obtained in a similar situation. This diameter reduction was less than $5 \mu \mathrm{m}$ and was therefore neglected in the data reduction process, since the uncertainties linked to the positioning of the measurement points caused by the random motions of the droplet were of the same order of magnitude.

Between the extreme measurement locations, the mean droplet temperature evolved from $35.4^{\circ} \mathrm{C}$ to $42.4^{\circ} \mathrm{C}$ in $4.3 \mathrm{~ms}$ (Fig. 11), which revealed strong heat transfer from the gaseous phase to the liquid droplet. The six temperature maps constructed at the different instants are shown in Fig. 12. In light of the experimental temperature maps, it was clearly observed that the heat diffusion within the droplet did not occur radially; the heating was mainly detected in the wake region, in qualitative agreement with the numerical simulations performed by Chiang et al. (1992). These numerical simulations, performed for cold $n$-octane droplets $(300 \mathrm{~K})$ suddenly injected in a hightemperature gas stream $(1250 \mathrm{~K})$ assume the formation of two Hill's vortexes inside the droplet because of the viscous friction between the liquid and the gas. The heat transfer from the hot gas environment occurs at the droplet surface, and the heated liquid fuel is then convected from the droplet surface to the wake side of the droplet and finally to the central region. The effect of the heat conduction tends also to homogenize the temperature between the hot and cold zones. All these phenomena can be observed in both present experimental results and in numerical simulations of Chiang et al. (1992).

\section{8}

\section{Concluding remarks}

Two-color laser-induced fluorescence is a powerful technique that can provide valuable mean temperatures of flying fuel droplets in combusting conditions. The modification of the optical set-up by increasing the spatial resolution, the use of periodically generated droplets
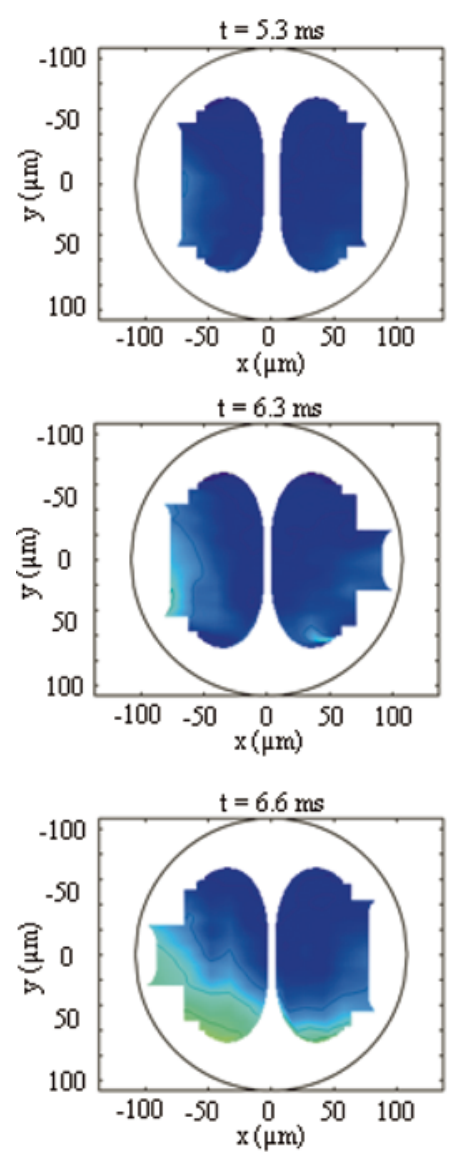
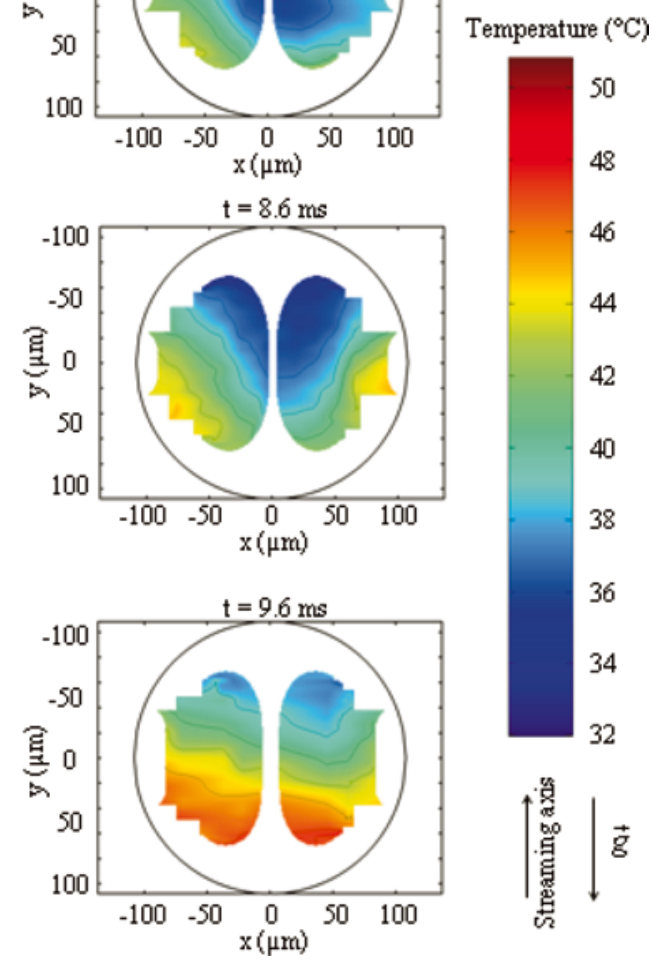

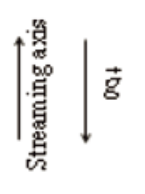

Fig. 12. Successive temperature maps of combusting ethanol droplets 
streaming on a linear trajectory and the development of an appropriate data reduction process enable us to measure temperature maps in a significant part of a droplet equatorial plane.

The absolute accuracy of the technique, according to the uncertainties linked to the calibration process and to the linearity of the detection chain, is estimated to $1^{\circ} \mathrm{C}$. The random error in the temperature in a temperature map can be estimated by observing a quasi-isothermal droplet temperature map: a random error of about $2^{\circ} \mathrm{C}$ is a good estimate. The space-resolved volume is roughly $20 \times 20 \times 57 \mu^{3}$, which is sufficient to give a good overview of the temperature gradients within the droplet. These measurements will be used to validate the heat diffusion models in moving droplets based on inside convective motions driven by Hill's vortexes combined with pure heat conduction. The more important problem is to give friction conditions at the air-droplet interface. It is expected that comparison of the experimental temperature fields with the numerical simulations will help in developing such conductive/convective models for the liquid fuel droplets injected in the combustion chambers.

\section{References}

Chiang CH, Sirignano WA (1993) Axisymmetric calculation of threedroplets interactions. Atomization Sprays 3:91-107

Chiang CH, Raju MS, Sirignano WA (1992) Numerical analysis of convecting, vaporizing, fuel droplet with variable properties. Int J Heat Mass Transfer 35:1307-1324

Copetta J, Rogers C (1998) Dual emission laser induced fluorescence for direct planar scalar behavior measurements. Exp Fluids 25: $1-15$

Gaskey S, Vacus P, David R, Villermaux J (1990) A method for the study of turbulent mixing using fluorescence spectroscopy. Exp Fluids 9:137-147
Lavieille P, Lemoine F, Lebouché M (2002) Experimental investigation on interacting low evaporating droplets temperature in linear stream using two colors laser induced fluorescence. Combust Sci Technol 174: 117-142

Lavieille P, Lemoine F, Lavergne G, Lebouché M (2001a) Evaporating and combusting droplet temperature measurements using two colors laser-induced fluorescence. Exp Fluids 31:45-55

Lavieille P, Lemoine F, Lebouché M (2001b) Combusting droplets temperature measurements using two colors laser-induced fluorescence: preliminary results and perspectives. CR Acad Sci Paris IIb 329:557-664

Lavieille P, Lemoine F, Lavergne G, Virepinte JF, Lebouché M (2000) Temperature measurements on droplets in monodisperse stream using laser-induced fluorescence. Exp Fluids 29:429-437

Lemoine F, Antoine Y, Wolff M, Lebouché M (1999) Simultaneous temperature and $2 \mathrm{D}$ velocity measurements in a turbulent heated jet using combined laser-induced fluorescence and LDA. Exp Fluids 26:315-323

Lu QZ, Melton LA (2000) Measurement of transient temperature field within a falling droplet. AIAA J 38:95-101

Mulholland JA, Srivastava RK, JOL Wendt (1988) Influence of droplet spacing on drag coefficient in nonevaporating, monodisperse stream. AIAA J 26:1231-1237

Muray AM, Melton LA (1985) Fluorescence methods for determination of temperature in fuel sprays. Appl Opt 24:2783-2787

Poo JY, Ashgriz N (1991) Variation of drag coefficient in an interacting drop stream. Exp Fluids 11:1-8

Sazhin SS, Dombrovsky LA, Krutitski P, Sazhina EM, Heikal MR (2002) Analytical and numerical modelling of convective and radiative heating of fuel droplets in diesel engines. In: Proc international heat transfer conference, 18-23 August 2002, Grenoble, France (on CD)

Silverman MA, Dunn-Rankin D (1994) Experimental investigation of a rectilinear droplet stream flame. Combust Sci Technol 100:57-73

Virepinte JF, Biscos Y, Lavergne G, Magre P, Collin G (2000) A rectilinear droplet stream in combustion: droplet and gas phase properties. Combust Sci Technol 150:143-159

Wells MR, Melton LA (1990) Temperature measurements of falling droplets. J Heat Transfer 112:1008-1013

Winter M, Melton LA (1990) Measurement of internal circulation in droplet using laser-induced fluorescence. Appl Opt 29:45744577 